\title{
Monodromy and the Structure of the Energy Spectrum of Hydrogen in Near Perpendicular Electric and Magnetic Fields
}

Christopher Robert Schleif

William \& Mary

John B. Delos

William \& Mary, jbdelos@wm.edu

Follow this and additional works at: https://scholarworks.wm.edu/aspubs

Part of the Physics Commons

\section{Recommended Citation}

Schleif, Christopher Robert and Delos, John B., Monodromy and the Structure of the Energy Spectrum of Hydrogen in Near Perpendicular Electric and Magnetic Fields (2007). Physical Review A, 76(1). https://doi.org/10.1103/PhysRevA.76.013404

This Article is brought to you for free and open access by the Arts and Sciences at W\&M ScholarWorks. It has been accepted for inclusion in Arts \& Sciences Articles by an authorized administrator of W\&M ScholarWorks. For more information, please contact scholarworks@wm.edu. 


\title{
Monodromy and the structure of the energy spectrum of hydrogen in near perpendicular electric and magnetic fields
}

\author{
Christopher R. Schleif and John B. Delos \\ Department of Physics, The College of William and Mary, Williamsburg, Virginia 23187-8795, USA
}

(Received 20 February 2007; published 12 July 2007)

\begin{abstract}
We examine the energy spectrum of hydrogen in weak near-perpendicular electric and magnetic fields using quantum computations and semiclassical analysis. The spectral structure is displayed in a lattice constructed by plotting the difference between total energy and first order energy versus first order energy, for all states of a given principal quantum number $n$. We have used classical perturbation theory to derive three approximate constants of the motion. Quantization of the resulting approximate actions produces a corresponding lattice in agreement with our quantum calculations. For some field arrangements, one of the actions is an intrinsically multivalued function of the constants of the motion. This produces defects in the lattices and signifies the presence of a classical phenomenon called monodromy. The presence of monodromy in the case of exactly perpendicular fields was previously predicted by Sadovskií and Cushman. Our quantum calculations and independent semiclassical analysis confirm their prediction, and show that the presence of monodromy not only persists into near-perpendicular fields, but also assumes other manifestations over a wider range of field strength ratios. We find that in near-perpendicular fields the structure of the spectrum is divided into six distinct parameter regions, which we characterize by the presence and type of lattice defect.
\end{abstract}

DOI: 10.1103/PhysRevA.76.013404

PACS number(s): 32.60.+i, 31.15.Gy, 32.30.-r

\section{INTRODUCTION}

The first order spectrum of the hydrogen atom in crossed electric and magnetic fields was predicted roughly 80 years ago by Pauli [1] and relatively recently explored in experiments [2]. Many theoretical investigations of this system have been published [3] and much attention has been given to the spectrum at higher order [4]. The hydrogen atom in applied fields has long been a model system for the study of order and chaos in classical and quantum mechanics, because it has just two or three degrees of freedom and a collection of controllable parameters and because it is accessible to both theory and experiment.

Meanwhile, a number of scientists have been studying a global property of integrable classical systems called "monodromy" [5]. The presence of monodromy in a classical integrable system may be identified by an intrinsic multivaluedness of classical action-angle variables [6]. After Duistermaat pointed out the existence of monodromy [5], Cushman and Bates identified several mechanical systems possessing monodromy, including the spherical pendulum and the Lagrange top [5]. Quantum manifestations of classical monodromy have been examined by several authors [7] including Child [7], San Vũ Ngoc [7], and Zhilinskií [7] and are commonly referred to as "quantum monodromy." Since in integrable systems, quantum eigenvalues correspond to quantized values of classical action variables, it follows that if an action variable is multivalued, then the corresponding quantum number is ambiguous. The presence of quantum monodromy may be detected by defects in a lattice constructed from energy eigenvalues.

These two lines of research were brought together by Sadovskií and Cushman [8]: By carrying out a classical perturbation calculation of hydrogen in perpendicular fields, they obtained an integrable approximation and showed that this system has monodromy.
Our purpose here is not to show or explain any of this theory, but to present the results of quantum computations of the spectrum of hydrogen in near-perpendicular fields along with semiclassical predictions made from an independent classical perturbation theory.

\section{BACKGROUND: PERPENDICULAR FIELDS}

We consider the quantum energy spectrum of a nonrelativistic, spinless hydrogen atom in static electric and magnetic fields $\mathbf{F}$ and $\mathbf{B}$. Let the $\mathbf{B}$ field vector define the $z$ axis and let the $\mathbf{F}$ and $\mathbf{B}$ field vectors together define the $x-z$ plane with $\chi$ denoting the angle between $\mathbf{F}$ and the $x$ axis. Then for weak, nearly perpendicular fields [9] the Hamiltonian may be written in the following ordering (atomic units):

$$
\begin{aligned}
\hat{H}= & \hat{H}_{0}+\hat{H}_{1}+\hat{H}_{2}=\underbrace{\frac{1}{2} p^{2}-\frac{1}{r}}_{\hat{H}_{0}}+\underbrace{\frac{1}{2} B L_{z}+F x}_{\hat{H}_{1}} \\
& +\underbrace{F x(\cos \chi-1)+F z \sin \chi+\frac{1}{8} B^{2}\left(x^{2}+y^{2}\right)}_{\hat{H}_{2}} .
\end{aligned}
$$

To obtain a collection of quantum spectra we expand the wave function in a basis [10] of spherical eigenstates of $H_{0}$, and compute eigenvalues $E_{j}$ of the resulting matrix [Fig. 1(a)]. The structure of the energy spectra may be summarized as follows: (i) The $n$ manifolds, as well as a further residual near degeneracy, are clearly distinguishable [see Fig. 1(a)]. (ii) Each $n$ manifold is split into $2 n-1$ equally spaced clusters of levels symmetrically displaced about $E_{o}$ $=-1 / 2 n^{2}$. (iii) The spacing between clusters depends upon the field strengths, and upon $n$ in proportion to 


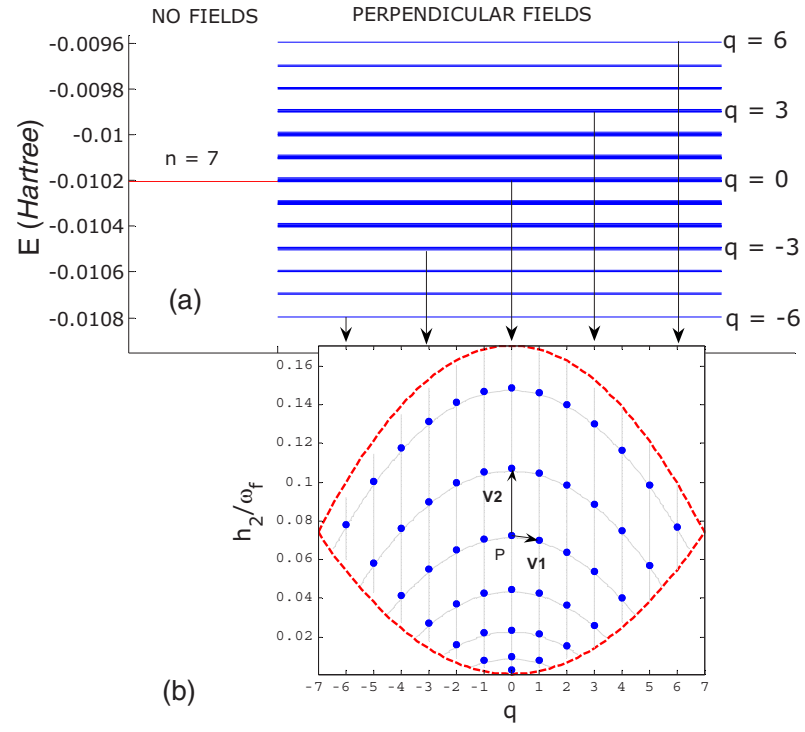

FIG. 1. (Color online) (a) The effects of perpendicular fields on the energy spectrum of a spinless hydrogen atom at principal quantum number $n=7$ for electric field $F=26.5 \mathrm{kV} / \mathrm{cm}$ and magnetic field $B=40 \mathrm{~T}$ or $\theta=32.8^{\circ}$ and $\omega_{f}=10^{-4}$ ( $\omega_{f}$ has the units of a magnetic field and is reported in atomic units in all figures). The quantum basis included $n$ manifolds $1-13$. When the fields are turned on, the $n^{2}$-degenerate $n$ manifolds are split into $2 n-1$ equally spaced $q$ manifolds each containing $n-|q|$ levels. The difference between an energy level and the energy of its parent $q$ manifold is denoted $h_{2}$. (b) Structure in the second order energies in an $n$ manifold is made visible by plotting $h_{2}$ versus $q$ (in all lattice figures we plot $h_{2} / \omega_{f}$ with units of energy over magnetic field reported in atomic units). Contours of quantized values of classical actions are plotted in gray and form a smooth grid organizing all lattice points. $\mathbf{V}_{2}$ extends from $P$ to the next lattice point along a contour of $q . \mathbf{V}_{1}$ extends to the nearest point along the curved contour. One may transport the pair $\left\{\mathbf{V}_{1}, \mathbf{V}_{2}\right\}$ one unit cell by simultaneously sliding the heads and tails along a set of contours until they each occupy the neighboring lattice point. For transport about any closed path inside the dashed boundary, the lattice vectors return to themselves, indicating a completely regular lattice.

$$
\omega_{f} \equiv \sqrt{B^{2}+(3 n F)^{2}} / 2 .
$$

(iv) Each cluster can be labeled by an integer quantum number $q$, where $-(n-1) \leq q \leq(n-1)$ such that the energy of each cluster (henceforth referred to as a $q$-manifold) is given by $E_{q}=E_{o}+q \omega_{f}$. (v) Each $q$ manifold contains exactly $n$ - $|q|$ states [see Fig. 1(b)].

These properties suggest that one may write each energy eigenvalue $E_{j}$ as the sum of energies of the $n$ manifold, $q$ manifold, and a small remaining term denoted $h_{2}$. Then,

$$
E_{j}=E_{n, q ; h_{2}}=-\frac{1}{2 n^{2}}+\omega_{f} q+h_{2} .
$$

The quantity $\omega_{f}$ defined in Eq. (2) is considered to be an effective field strength characterizing the magnitude of the combined field perturbation such that $\left|h_{2}\right| \sim \omega_{f}^{2}$. The following definition of $\theta$ allows a convenient parametrization of all possible field magnitude ratios at a given perturbation strength $\omega_{f}$ :

$$
\theta \equiv \tan ^{-1}(3 n|F| /|B|), \quad 0 \leq \theta \leq \pi / 2 .
$$

This implies $F=2 \omega_{f} \sin (\theta) / 3 n$ and $B=2 \omega_{f} \cos \theta$ with the Zeeman and Stark limits at $\theta=0$ and $\theta=\pi / 2$, respectively. The main utility of this parametrization is that the characteristic shape and features of each spectral lattice are stationary with respect to $n$ at fixed $\theta$ and fixed scaled angle $\chi / \omega_{f} n^{3}$. Changing $n$ only changes the number of lattice points which populate the fixed structure.

To investigate the structure present in the second order corrections $h_{2}$, it is useful to plot the value of $h_{2}$ versus $q$. The resulting plot is a two-dimensional lattice consisting of $n^{2}$ points, which is called the second-order quantum energymomentum spectrum (also known as the joint spectrum or simply the second-order spectrum; see Fig. 1).

Looking at only the lattice points in Fig. 1(b), it is obvious to the eye that there is structure hidden "in between the points." Along with the spectrum are plotted several structures we obtained from classical perturbation theory. The lattice has a continuous boundary depicted by the dashed curves. By construction all points lie on vertical lines of constant $q$. Most important, all energies can be organized into smooth curves transverse to the vertical lines. In fact, in the classical perturbation theory, $n, q$, and the smooth curves in Fig. 1 are all quantized values of classical action variables. These actions are smooth and single valued functions of $q$ and $h_{2}$, defining a single smooth coordinate system covering all points inside the spectral boundary [11]. Any spectral lattice with this property is said to form a completely regular lattice. If the spectrum in Fig. 1(b) were transformed into the smooth action coordinates one would find that all points lie on contiguous intersections of a rectangular grid.

The "trivial" nature of this lattice can be characterized by a matrix, which represents the effect of the transport of any pair of independent lattice vectors around any closed loop inside the spectral boundary. The lattice vectors $\left\{\mathbf{V}_{1}, \mathbf{V}_{2}\right\}$ at $P$ [Fig. 1(b)] are unchanged by such transport, so that the matrix is the unit matrix $E=[1,0 ; 0,1]$. Any such completely regular spectrum is completely described by the singleelement list of matrices $\{E\}$.

In Fig. 1 we have carefully chosen critical values of $\theta$ and $\chi$ to obtain the completely regular spectrum. Not all values of $\theta$ and $\chi$ produce a regular lattice. Among the ways a hydrogen spectral lattice can fail to be totally regular are the following, discovered by Sadovskií and Cushmann [8] in the case of exactly perpendicular fields $\chi=0$. (i) There are ranges of $\theta$ at $\chi=0$ such that the spectrum is divided into two disjoint trivial subsections, with different smooth action coordinates in each section. These subsections are divided by boundaries similar to the outer boundary of the spectrum (here denoted with dashed curves). Examples of such disjoint spectra can be found at perpendicular fields for $\theta<\theta_{1}$ and $\theta>\theta_{2}$, where

$$
\theta_{1}=\cos ^{-1}\left(2^{-1 / 4}\right), \quad \theta_{2}=\cos ^{-1}\left(\sqrt{\frac{\sqrt{6}}{2}-1}\right) .
$$




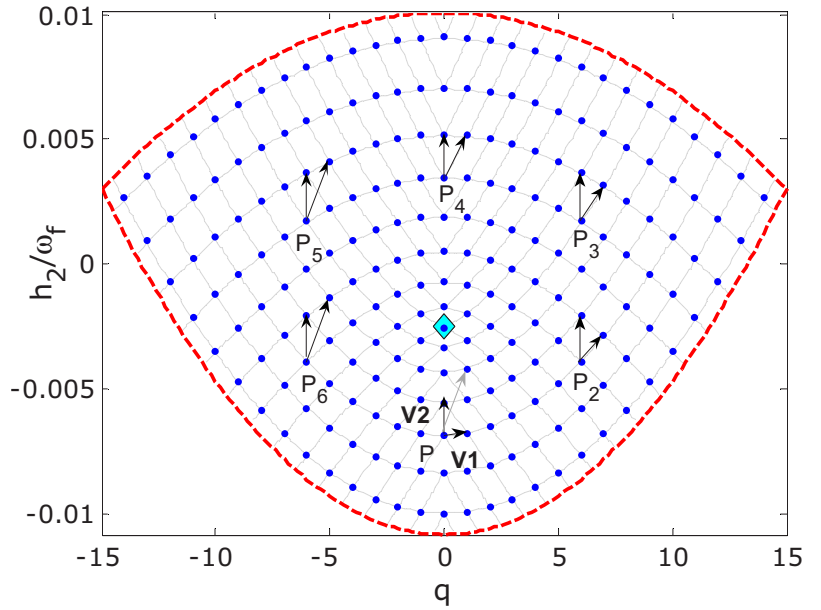

FIG. 2. (Color online) a similar diagram appears in Ref. [8]) The monodromy in perpendicular fields. The spectrum $h_{2}$ versus $q$ for $\chi=0 \mathrm{rad}, n=15, \quad F=175 \mathrm{~V} / \mathrm{cm}, B=0.302 \mathrm{~T}$ or $\theta=50^{\circ}$ and $\omega_{f}$ $=10^{-6}$. The quantum basis included $n$ manifolds $11-19$. Two families of gray contours are plotted. The upper family passes smoothly through $q=0$ above the diamond but has a discontinuous derivative at $q=0$ below the diamond. Similarly the lower family is only smooth below the diamond. A defect is characterized by lattice vector transport of $\left\{\mathbf{V}_{1}, \mathbf{V}_{2}\right\}$ along smooth contours connecting points marked by a sequence of $P$ 's. When a complete circuit has been made $\mathbf{V}_{1}$ returns to the gray vector $\mathbf{V}_{1}+2 \mathbf{V}_{2}$ indicating a $[1,0 ; 2,1]$ defect.

In such a case one could still characterize the spectrum by a single matrix $\{E\}$, which describes the effects of lattice transport within each separate region. (ii) For a certain range $\theta_{1}<\theta<\theta_{2}$ at $\chi=0$, Sadovskií and Cushman have shown the existence of a point defect which introduces nontrivial effects of lattice-vector transport, characterized by matrices other than the identity. The following paragraphs demonstrate how to associate a list of matrices with any such defective spectrum.

By construction, every spectrum at fixed $n$ has points on contours of constant $q$, so these contours are tacitly chosen as one good coordinate on the spectrum. Defects arise when one attempts to choose another smooth coordinate function independent of $q$. When a lattice defect is present, the spectrum admits only locally consistent choices of smooth action contours.

In Fig. 2 we have plotted two families of contours connecting the lattice points. Given only the lattice points, one might start at the top row and draw contours similar to the upper boundary through the lattice points and continue row by row downward [as in Fig. 1(b)]. After a certain point marked by the diamond, the contours can no longer be drawn smoothly through $q=0$, so they cannot be interpreted as level sets of a smooth action coordinate everywhere on the spectrum. Similarly, starting at the lower boundary, the lattice points are connected by smooth curves similar to the lower boundary only as far as the diamond marker. Neither of these choices can be used for smooth coordinates on the entire spectrum. However, one can find locally smooth contours in any region excluding the diamond by considering two such choices. The spectrum is said to be locally trivial, having a locally regular lattice, with a defect associated with the cyan diamond such that this spectrum requires at least two smooth action charts [12]. The point source of the defect, marked by the diamond, is called a monodromy center.

The local triviality allows a well defined notion of lattice vector transport for circuits on the spectrum. One may characterize the defect by transport around any loop encircling the diamond. In Fig. 2, begin at point $P$ and choose the lower family of contours as locally smooth contours connecting the lattice points in a region about $P . \mathbf{V}_{2}$ extends from lattice point $P$ to the next neighbor along a contour of constant $q$. $\mathbf{V}_{1}$ extends to the nearest neighbor along the curved contour of the lower family. Slide the heads and tails of these two vectors along locally smooth contours (including contours of constant $q$ ) into neighboring lattice points until an entire counterclockwise circuit about the diamond has been made. Transport of the two vectors around the entire counterclockwise circuit in Fig. 2 has the result that $\mathbf{V}_{2}$ comes back to itself $\mathbf{V}_{2} \rightarrow \mathbf{V}_{2}$ while $\mathbf{V}_{1}$ comes back to a different vector which is $\mathbf{V}_{1} \rightarrow \mathbf{V}_{1}+2 \mathbf{V}_{2}$. This transformation is equivalent to multiplication by the matrix $M_{2}=[1,0 ; 2,1]$ written in the lattice basis $\left\{\mathbf{V}_{1}, \mathbf{V}_{2}\right\}$ at $P$ :

$$
M_{2}=\left[\begin{array}{ll}
1 & 0 \\
2 & 1
\end{array}\right], \quad\left\{\mathbf{v}_{1}, \mathbf{v}_{2}\right\}=\left\{\left[\begin{array}{l}
1 \\
0
\end{array}\right],\left[\begin{array}{l}
0 \\
1
\end{array}\right]\right\} .
$$

This $M_{2}$ defect was predicted by Sadovskií and Cushman for perpendicular fields.

If vectors are transported about any "trivial loop" in Fig. 2 (one which does not encircle the diamond) one recovers the transformation $E$. Thus there are two independent counterclockwise circuits present in the spectrum in Fig. 2. They act as a basis for any circuit on the spectrum. For example, a circuit consisting of twice around the diamond and once about a trivial loop results in the transformation $M_{2} M_{2} E$ $=[1,0 ; 4,1]=M_{4}$, which may also be obtained by the obvious matrix multiplication. Also, each counterclockwise circuit has an inverse which is the same curve traversed in the opposite sense. For example, if the circuit in Fig. 2 is traversed in a clockwise sense, the result is the transformation $M_{2}^{-1}=[1,0 ;-2,1]=M_{-2}$.

Thus, to each quantum spectrum is associated a list of matrices, one for each basis circuit. The spectrum in Fig. 1 belongs to a family of spectra which are characterized by the single-element list $\{E\}$, while spectra like that in Fig. 2 would be characterized by a two-element list $\left\{E, M_{2}\right\}$.

\section{RESULTS: NEAR-PERPENDICULAR FIELDS}

We extend the earlier discoveries by considering the case of nearly perpendicular fields, such that $|\chi| / \omega_{f} n^{3}<1$, at all electric and magnetic field ratios $\theta$. Much of the organization of the spectrum at perpendicular fields is retained into the near-perpendicular region; in particular, the $n$ and $q$ clustering of the energy levels remains intact, allowing the same construction of spectral lattices. However, the second-order structure of the spectrum changes considerably. The effect of nonzero $|\chi|$ is to introduce several classes of spectra characterized by different lattice defects. 


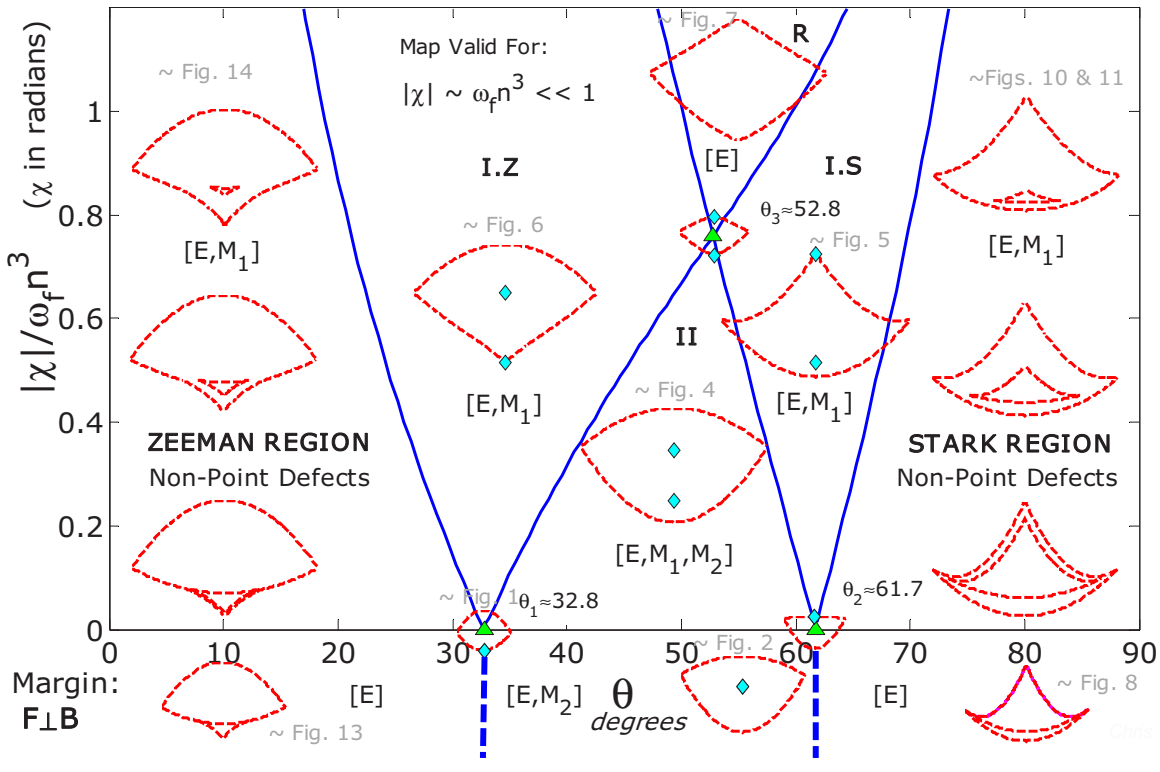

FIG. 3. (Color online) A map of spectral structure and lattice defects for all near-perpendicular field configurations. All field magnitude ratios are covered on the horizontal axis by Eq. (4) with $0^{\circ} \leq \theta \leq 90^{\circ}$, with pure magnetic field at $\theta=0^{\circ}$ and pure electric field at $\theta=90^{\circ}$. $\pi / 2-\chi$ is the angle in radians between the electric and magnetic fields, with perpendicular fields $\chi=0$ along the lower margin of the map. For $0<|\chi| \sim \omega_{f} n^{3} \ll 1$, the lines demarcate six regions with distinct spectral types. In each region, depictions of the classical structure of typical spectra appear along with a bracketed list of matrices indicating the quantum spectral lattice classification. The matrices describe the effects of lattice vector transport about closed circuits.

Using classical perturbation theory we have reduced the full classical Hamiltonian (1) to a one-degree-of-freedom effective Hamiltonian defined on a surface having the topology of a sphere. For $\omega_{f} n^{3} \ll 1$, contours of this effective Hamiltonian give an accurate description of the structure of the spectrum, including the sources of lattice defects, and quantization of action for this effective Hamiltonian gives an accurate approximation for almost all eigenvalues [13].

Using this effective Hamiltonian, we have mapped out all lattice defects observable in the near perpendicular region. The results are shown in Fig. 3. The lines demarcate six regions in the parameter space. Each region contains a family of spectra with common lattice defect structure. A characteristic list of matrices and a plot of a typical spectrum's classical structure appear in each zone. The spectra and matrix lists appearing in the lower margin along the $\theta$ axis are for the case of exactly perpendicular fields $\chi=0$.

We examine each of the spectral structures in the following sections.

\section{A. Region II: Bifurcations from the perpendicular monodromy interval}

As discussed earlier, at perpendicular fields, for any $\theta$ such that $\theta_{1}<\theta<\theta_{2}$, the spectrum contains a single $M_{2}$ point defect (Fig. 2). As the fields are displaced from perpendicular by increasing $|\chi|$, this point bifurcates into two distinct $M_{1}$ $=[1,0 ; 1,1]$ defect points (Fig. 4).

$$
M_{1}=\left[\begin{array}{ll}
1 & 0 \\
1 & 1
\end{array}\right] .
$$

In Fig. 4 we mark two of the three possible nontrivial counterclockwise circuits by sequences of Latin and Greek letters. The transport of $\mathbf{V}_{1}$ and $\mathbf{V}_{2}$ about the sequence of Latin letters yields the $M_{2}$ defect while transport about the sequence of Greek letters yields $M_{1}$. There are no other independent nontrivial circuits, for if we traverse the Latin loop and then make a clockwise circuit about the Greek loop, the total circuit is equivalent to a single circuit about the lower diamond marker. Thus this spectrum can be classified by the list $\left\{E, M_{1}, M_{2}\right\}$. Similar spectra are obtained from any perpendicular spectrum in the interval $\theta_{1}<\theta<\theta_{2}$ by tilting $\chi$. We call this region II.

\section{B. Regions I.S and I.Z}

If we start with a spectrum from region II (Fig. 4) and continue to tilt the field angle $\chi$ at fixed $\theta$ (with $\theta_{1}<\theta<\theta_{2}$ ), then the defect points change energy until one (or both) reaches the boundary of the second-order spectrum. At larger $\theta$ (closer to Stark), the higher defect reaches the upper boundary first, while at smaller $\theta$ (closer to Zeeman), the lower defect reaches the lower boundary first. Both defects will join the boundary simultaneously at $\chi=\chi_{3}$ only if $\theta$ $=\theta_{3}$ where

$$
\chi_{3}= \pm \sqrt{\frac{\sqrt{3}-1}{3-\sqrt{3}}} \omega_{f} n^{3}, \quad \theta_{3}=\cos ^{-1}\left(\sqrt{\frac{\sqrt{3}}{2}-\frac{1}{2}}\right) .
$$

If $\theta_{3}<\theta<\theta_{2}$ then the upper defect point from region II joins the upper boundary to yield a spectrum with a single $M_{1}$ defect, which is therefore characterized by $\left\{E, M_{1}\right\}$. We call this region I.S. A representative spectrum is depicted in Fig. 5.

Another region with $\left\{E, M_{1}\right\}$ structure may be reached by starting in region II with $\theta_{1}<\theta<\theta_{3}$ and increasing $|\chi|$. In 


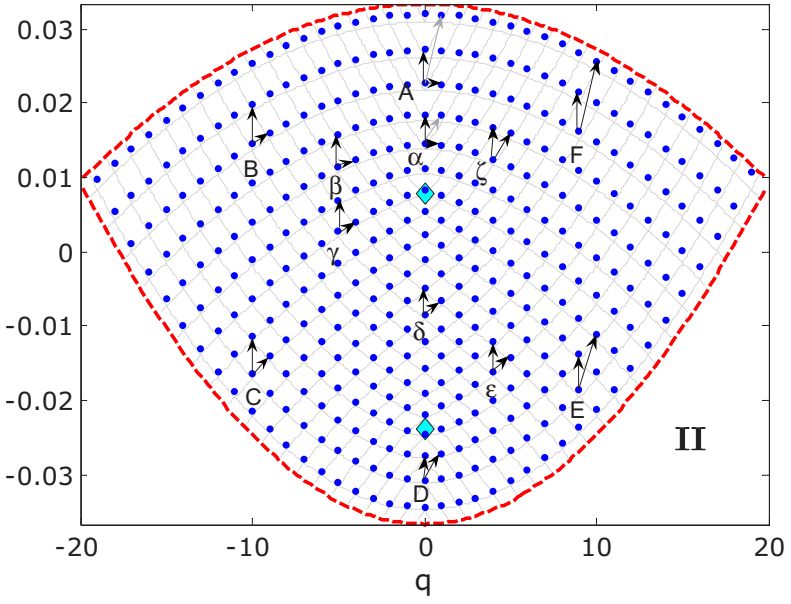

FIG. 4. (Color online) Type II spectrum. The spectrum $h_{2}$ versus $q$ for $\chi / \omega_{f} n^{3}=0.2, n=18, F=146 \mathrm{~V} / \mathrm{cm}, B=0.302 \mathrm{~T}$, or $\theta=50^{\circ}$ and $\omega_{f}=10^{-6}$. The quantum basis included $n$ manifolds $14-22$. The circular dots are derived from quantum calculations, while all other structures were derived from classical perturbation theory and semiclassical quantization of actions. This spectrum is obtained from one similar to Fig. 2 by tilting the fields from perpendicular. A point defect at perpendicular fields bifurcates into the two point defects shown above for nonzero $\chi$. Two independent closed contours are marked by sequences of letters. A $[1,0 ; 2,1]$ defect is characterized by lattice vector transport of $\left\{\mathbf{V}_{1}, \mathbf{V}_{2}\right\}$ along locally smooth action contours connecting points marked by the Latin letter cycle $A B C D E F A$. This circuit encircles both point defects and after a complete circuit has been made, $\mathbf{V}_{1}$ returns to the vector $\mathbf{V}_{1}+2 \mathbf{V}_{2}$ depicted in gray at $A$. A $[1,0 ; 1,1]$ defect is characterized by transport of $\left\{\mathbf{V}_{1}, \mathbf{V}_{2}\right\}$ along smooth contours connecting points marked by the sequence of Greek letters $\alpha \beta \gamma \delta \varepsilon \zeta \alpha$. When a complete circuit has been made $\mathbf{V}_{1}$ returns to the vector $\mathbf{V}_{1}+\mathbf{V}_{2}$ depicted in gray at $\alpha$.

this case, the lower defect from region II will join the lower boundary, again yielding a spectrum with a single $M_{1}$ defect.

Analogous transitions of the type II spectra occur when the field strength ratio $\theta$ is increased (II $\rightarrow \mathrm{I}$.S $)$ or decreased $\left(\mathrm{II} \rightarrow \mathrm{I}\right.$.Z) at fixed $\chi$ with $0<|\chi|<\chi_{3}$. These transitions occur at the values of $\theta$ marked by the lines demarcating region II from its neighboring regions on the map in Fig. 3. (See Fig. 6.)

\section{Completely regular region $R$}

If $\theta$ is exactly equal to $\theta_{3}$, we pass directly from a type-II spectrum to a completely regular spectrum by increasing $|\chi|$. We may also recover a completely regular spectrum from an I.S or I.Z type by varying $\theta$ and $|\chi|$ such that the single defect point is moved to the boundary of the spectrum. The values of $\theta$ and $|\chi|$ for which this occurs are marked on the map by the lines separating the I.S and I.Z regions from their neighbor at large $|\chi|$ (Fig. 3). For all spectra in the $R$ region, every closed lattice vector transport loop leaves the lattice vectors unchanged. We label this featureless region $R$ (for completely regular). Any spectrum in this region may be covered with a single action chart, and all $R$ spectra are classified by the list $\{E\}$. (See Fig. 7.)

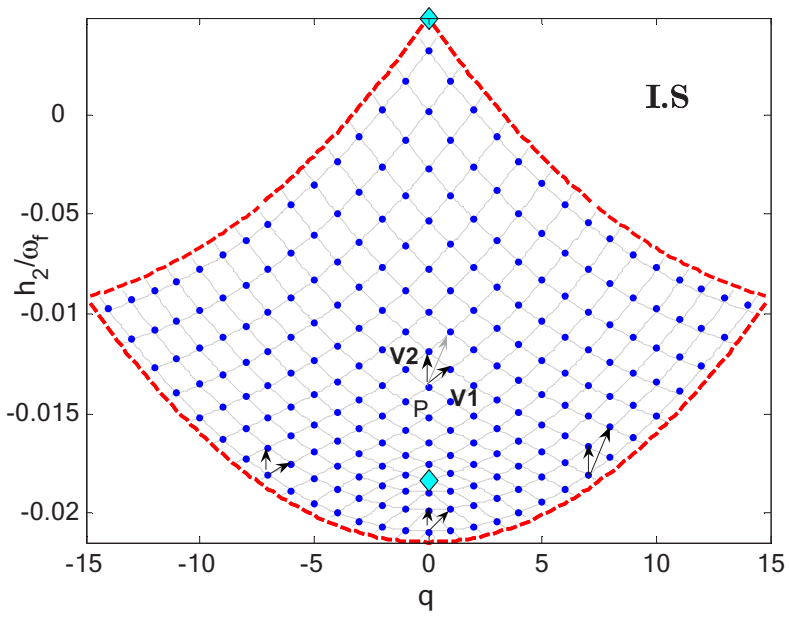

FIG. 5. (Color online) Type I.S spectrum. The spectrum $h_{2}$ versus $q$ for $|\chi| / \omega_{f} n^{3}=0.6, n=15, F=207 \mathrm{~V} / \mathrm{cm}, B=0.199 \mathrm{~T}$, or $\theta$ $=65^{\circ}$ and $\omega_{f}=10^{-6}$. In this calculation, and all calculations at $n$ $=15$, the quantum basis included $n$ manifolds 11-19. By considering the effects of lattice vector transport a type I.S spectrum would be classified $\left\{E, M_{1}\right\}$. This spectrum may be obtained from a type II spectrum with $\theta_{3}<\theta<\theta_{2}$ by increasing $|\chi|$ at constant $\theta$. At a critical $|\chi|$, the upper point defect from a region II spectrum joins the upper boundary leaving a single $M_{1}$ defect.

\section{Stark region: Bifurcations from perpendicular fields near the Stark limit}

The spectra in the Stark region possess a more complex structure. To understand the behavior here, we first reexamine the second order structure in perpendicular fields, previously explained by Solov'ev [3(a)] but using our semiclassical framework. For perpendicular fields the second order spectrum consists of three families of states (Fig. 8). States at low energy are nondegenerate, while states at high energy

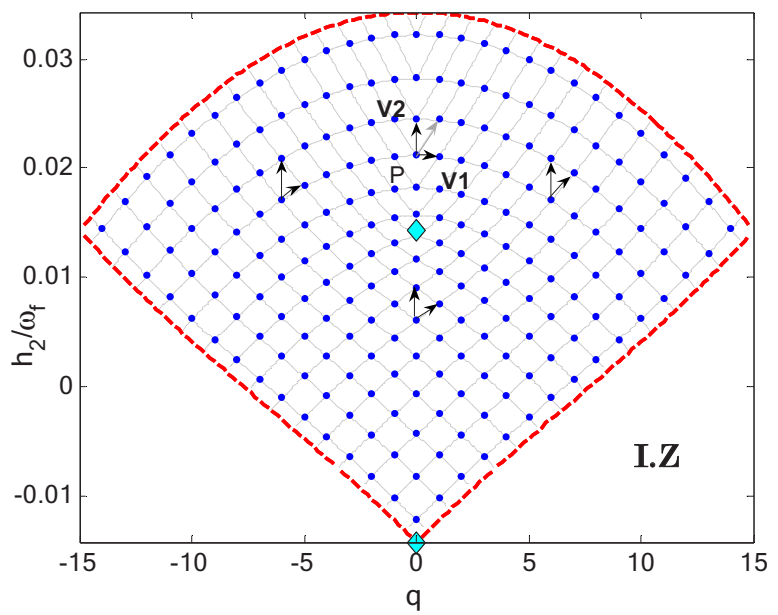

FIG. 6. (Color online) A type I.Z spectrum. The spectrum $h_{2}$ versus $q$ for $|\chi| / \omega_{f} n^{3}=0.6, n=15, F=131 \mathrm{~V} / \mathrm{cm}, B=0.385 \mathrm{~T}$, or $\theta=35^{\circ}$ and $\omega_{f}=10^{-6}$. This spectrum may be obtained from one similar to Fig. 4 with $\theta_{1}<\theta<\theta_{3}$ by increasing $|\chi|$. At a critical $|\chi|$, the lower point defect from the type II spectrum joins the lower boundary of the spectrum. The upper $[1,0 ; 1,1]$ defect remains present. 


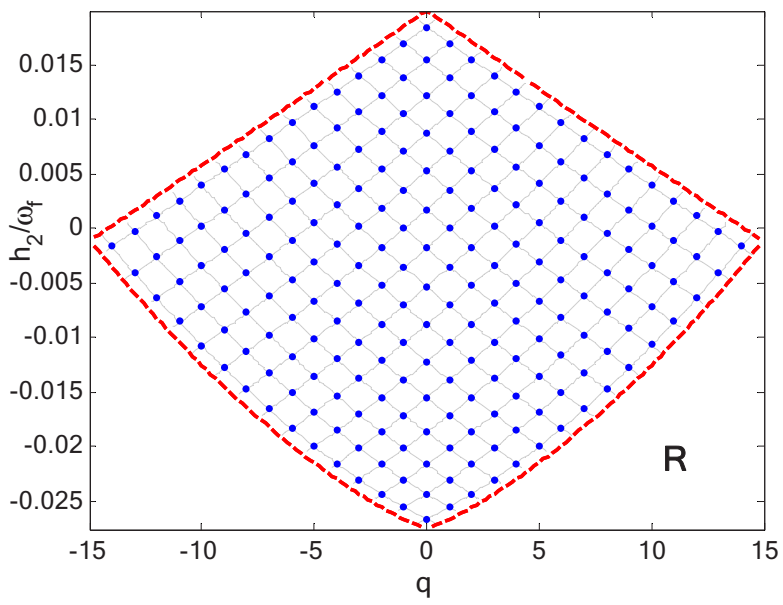

FIG. 7. (Color online) A type $R$ spectrum. The spectrum $h_{2}$ versus $q$ for $|\chi| / \omega_{f} n^{3}=1, n=15, F=187 \mathrm{~V} / \mathrm{cm}, B=0.27 \mathrm{~T}$, or $\theta$ $=55^{\circ}$ and $\omega_{f}=10^{-6}$. Spectra in the $R$ region are completely regular and admit a single smooth action chart. An $R$ spectrum may be obtained from a type II spectrum with $\theta=\theta_{3}$, by increasing $|\chi|$, or from a type I.S or I.Z spectrum by varying $(\theta,|\chi|)$ along a path which crosses their respective upper borders as indicated by the lines on the map in Fig. 3.

are very nearly doubly degenerate. The degenerate states are separated from the nondegenerate states by a classical boundary.

To understand this structure let us discuss the reduced Hamiltonian $h_{2}$ we derived from classical perturbation theory (mentioned at the beginning of part II). The reduced Hamiltonian is a $\pi$-periodic function of an angle $\delta_{w}\left(-\pi / 2<\delta_{w}\right.$ $\leq \pi / 2$ ), and its conjugate momentum $W$. It also depends parametrically on the classical principal action $N=n \hbar$ and a

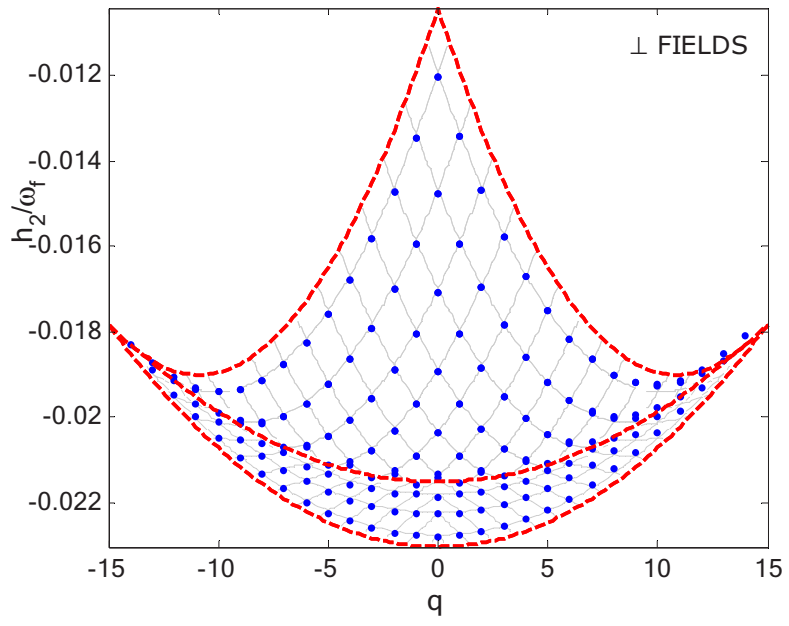

FIG. 8. (Color online) A perpendicular fields spectrum near the Stark limit. The spectrum $h_{2}$ versus $q$ for $\chi=0, n=15, F$ $=225 \mathrm{~V} / \mathrm{cm}, B=0.082 \mathrm{~T}$, or $\theta=80^{\circ}$ and $\omega_{f}=10^{-6}$. Spectra with $\chi$ $=0$ and $\theta_{2}<\theta<90$ are composed of two disjoint regular regions. All quantum states in the upper triangular region are doubly degenerate with a slight splitting due to tunneling, while all states in the lower crescent shaped region are nondegenerate. The boundary between the two regions marks the energy of a saddle point in the reduced second order Hamiltonian $h_{2}$ at each $Q=q$.
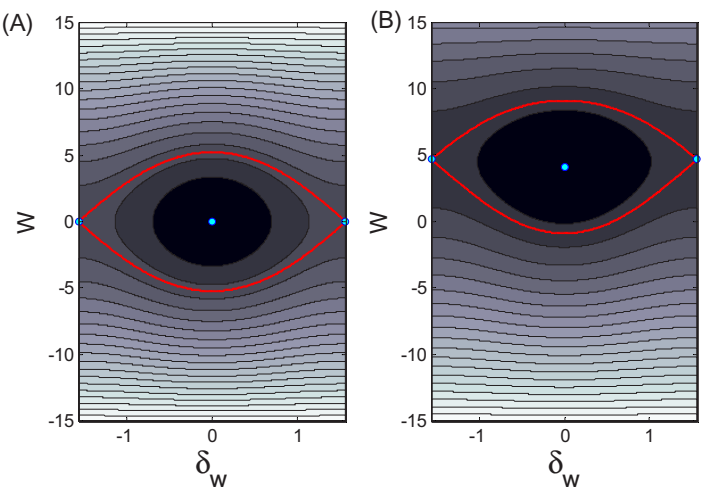

FIG. 9. (Color online) Contours of the reduced second order Hamiltonian $h_{2}\left(W, \delta_{w} ; n, Q\right)$ at $n=15, Q=0$, in the Stark region with $\theta=80^{\circ}$ and (a) $\chi=0$ at perpendicular fields and (b) $\chi / \omega_{f} n^{3}=0.8$. This Hamiltonian is a function on a spherical phase space such that $W$ is related to a latitude and $\delta_{w}$ to a longitude. The contour plots are then to be interpreted as a planar projection of the reduced phase space, with left and right edges identified and top and bottom boundaries corresponding to north and south poles, respectively. Darker regions indicate lower energies; points indicate fixed points and an $x$-point separatrix is marked. In addition to the $o$ point in the center of the dark region, two additional $o$ points are present but not marked at the north and south poles. The separatrix divides the classical contours into three families of states. (A) At perpendicular fields the reduced Hamiltonian is symmetric about the equator $W$ $=0$. At low energies are the "equatorial" states localized about $W$ $=0$. At high energies, "northern" states are localized at positive $W$, and "southern" states are localized at negative $W$. (B) When the fields are tilted the symmetry is broken such that both the phase space area and the energy range occupied by the northern states are reduced, while those of the southern states are increased. The structure of the contours of $h_{2}$ is similar for all Q less than a certain $Q_{\text {critical }}(N, \theta, \chi)$.

classical action variable $Q=q \hbar$ corresponding to the $q$ clustering integer $(\hbar=1)$. In the reduced system, $N$ and $Q$ are both constants of the motion. For each $|Q|<N$, the reduced phase space $\left(W, \delta_{w}\right)$ has the topology of the surface of a sphere with $W$ related to a latitude and $\delta_{w}$ related to a longitude. The values of this Hamiltonian describe the classical second order energy and its derivatives give the motion of the system point on the surface of the sphere. The maximum and minimum values that $h_{2}$ assumes on the sphere (at a given $N=n, Q=q$ ) are the upper and lower dashed boundaries that encase all the quantum spectra plotted in this paper.

Contour plots of this reduced Hamiltonian in the Stark region are shown in a planar projection in Figs. 9(a) and 9(b) for the case $N=15, Q=0$ and (a) perpendicular fields, (b) $\chi$ $=0.8 n^{3} \omega_{f}$. Darker regions indicate lower energies, markers indicate fixed points of the Hamiltonian, and the contour is a separatrix having the energy of an $x$ point. The separatrix divides the classical phase space structure into three families of energy level sets. At perpendicular fields (a), the Hamiltonian is symmetric about the "equator" $W=0$. The low energy family is localized in $W$ symmetrically about the equator and so we refer to this family as the equatorial family. The two high energy families are localized in $W$ in either the northern $(W>0)$ or southern $(W<0)$ hemispheres, so we 
refer to them as the "northern" or "southern" families, respectively.

Using a primitive semiclassical approximation, quantum states are associated with contours having half-integral values of an action variable related to the $\left(W, \delta_{w}\right)$ phase space area [14]. At perpendicular fields, the reduced Hamiltonian is always symmetric in $W$. Inspection of Fig. 9(a) reveals that all level sets with energies greater than that of the $x$ point appear as disjoint pairs of contours, one in the north and one in the south. If a northern contour with energy $h_{2}=E_{2}$ has a quantized value of the action variable, then from the $W$ symmetry, it follows that there is a southern contour with the same energy and action value. Thus, at all allowed energies greater than that of the separatrix, we expect to find two nearly degenerate quantum states. At each energy below the separatrix, there exists only a single closed contour so we expect the corresponding quantum states to be nondegenerate. This three-family structure, due to the presence of the $x$ point, exists in the contours of the reduced Hamiltonian for all $|Q|$ less than a certain critical value $Q_{\text {critical }}(N, \theta, \chi)$. Therefore, we expect to find the quantum degeneracy implied by such a structure for all $|q|<Q_{\text {critical }}(N=n, \theta, \chi)$.

Looking back at Fig. 8 one identifies the nondegenerate states in the lower crescent as the equatorial states. The boundary between the crescent and triangular regions marks the energy of the $x$-point separatrix on each reduced phase space having $|Q|<Q_{\text {critical }}\left(N=15, \theta=80^{\circ}, \chi=0\right)=14.06$. The upper triangular region is filled with pairs of nearly degenerate states, with slight splitting, greatest near the $x$-point energy. This small splitting arises from quantum tunneling, so the resulting states are symmetric and antisymmetric combinations of northern and southern states.

When the angle between the fields is changed, the symmetry in the Hamiltonian between the northern and southern hemispheres is broken. If we start with the contours in Fig. 9(a) and tilt the fields from perpendicular, we introduce a small term proportional to $-\chi W$, producing contours like those plotted in Fig. 9(b) at $\chi=0.8 n^{3} \omega$. By comparing these contours with those in Fig. 9(a) we see that the energy range and phase space area occupied by southern states increased while the energy range and phase space area of the northern states decreased.

Since each quantum state corresponds to an energy contour having a quantized action, we expect to find in the spectrum fewer northern states occupying a smaller energy range and more southern states occupying a larger energy range [15]. There is similar behavior for all $|q|<Q_{\text {critical }}$. Stark region spectra with tilted fields are shown in Figs. 10 and 11. The southern states are associated with the gray action contours which occupy the whole energetically allowed region of the spectrum. The northern states are associated with the action contours which are confined to the smaller triangular region. The lower boundary of this triangle marks the energy of the $x$ point for each $|Q|$ less than $Q_{\text {critical }}(n, \theta, \chi)$. The upper boundary is the maximum energy of the northern contours. Therefore, the gray action contours associated with the southern states pass smoothly through the upper boundary. Neither northern nor southern actions can be smoothly continued through the $x$-point (lower) boundary of the inner triangle.

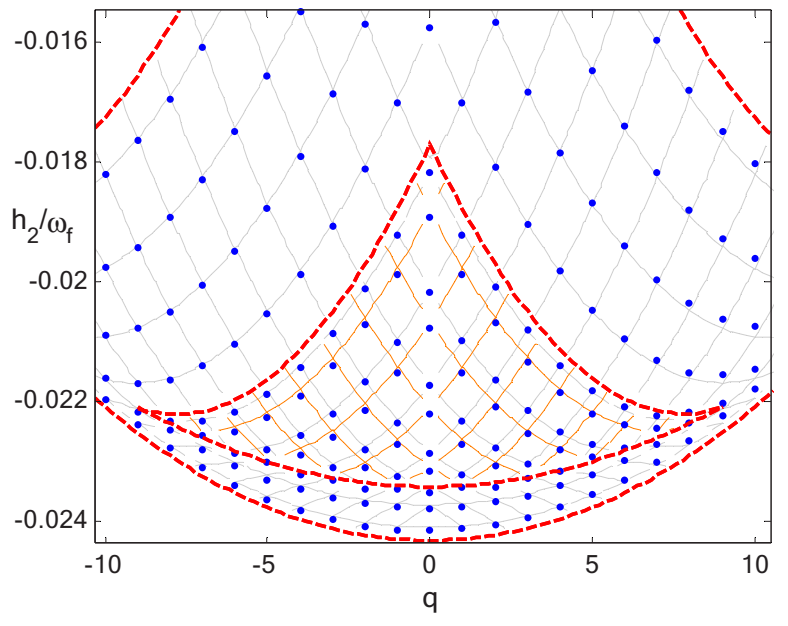

FIG. 10. (Color online) A portion of a Stark region spectrum; $h_{2}$ versus $q$ for $|\chi| / \omega_{f} n^{3}=1, n=15, F=226 \mathrm{~V} / \mathrm{cm}, B=0.0654 \mathrm{~T}$, or $\theta$ $=82^{\circ}$ and $\omega_{f}=10^{-6}$. A triangular subarea of all Stark region spectra with $|\chi|>0$ contains two families of states with overlapping energy ranges. "Southern" states occupy the entire allowed region of the spectral plane, and their action contours are light gray. "Northern" states occupy only the inner triangular region, and their action contours are heavier (orange online). Neither northern nor southern action contours pass smoothly through the lower boundary of the inner region.

In contrast to the orange contours, which represent a single valued action variable, the gray curves represent an intrinsically multivalued action. This is evident in Fig. 11 where we consider the effects of lattice vector transport along gray action contours on a closed loop. We obtain an $M_{1}$ lattice defect whenever the lower boundary of the small triangular region is enclosed by a single counterclockwise circuit.

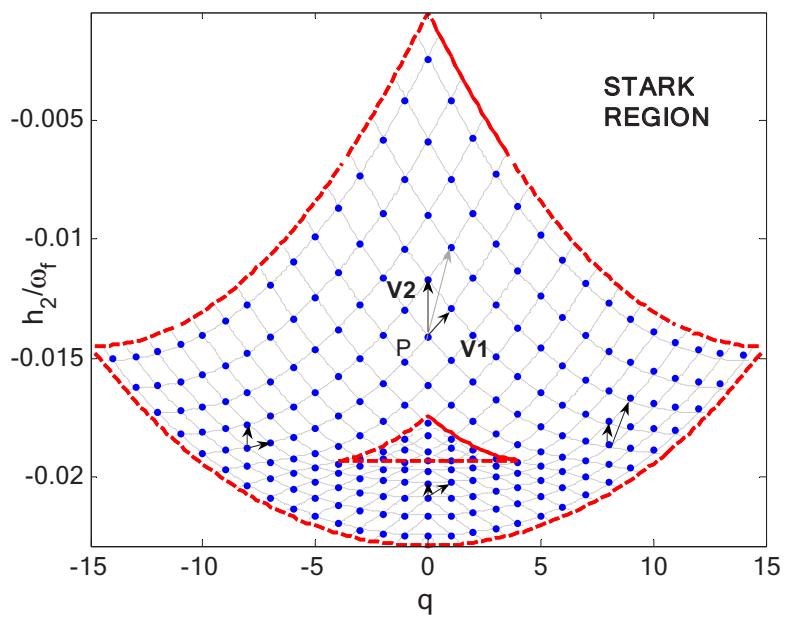

FIG. 11. (Color online) Stark region. The spectrum $h_{2}$ versus $q$ for $|\chi| / \omega_{f} n^{3}=0.6, n=15, F=219 \mathrm{~V} / \mathrm{cm}, B=0.137 \mathrm{~T}$, or $\theta=73^{\circ}$ and $\omega_{f}=10^{-6}$. All Stark region spectra with $|\chi|>0$ contain a smaller triangular region containing two families of states. The lower boundary of the smaller triangular region acts as a defect source. Lattice vector transport about any counterclockwise closed loop encircling the lower boundary of the inner triangle displays the presence of an $M_{1}$ defect. 

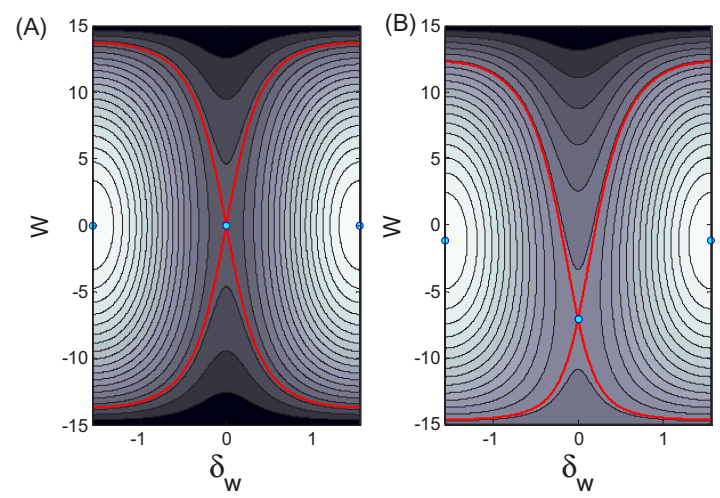

FIG. 12. (Color online) Contours of the reduced second order Hamiltonian $h_{2}(W, \delta w)$ at $n=15, Q=0$, in the Zeeman region where $\theta=15^{\circ}$ and (a) perpendicular fields $(\chi=0)$ and (b) $\chi / \omega_{f} n^{3}=0.7$. Darker regions indicate lower energies; points indicate fixed points and an $x$-point separatrix is marked. There are also unmarked $o$ points located at the north and south poles of both spheres. The separatrix divides the classical contours into three families of states. At high energies are the "equatorial" states, and at low energies are the "northern" states at positive $W$ and "southern" at negative $W$. (a) At perpendicular fields the Hamiltonian is symmetric about the equator $W=0$. (b) When the fields are tilted the symmetry is broken such that both the phase space area and the energy range occupied by the southern states are decreased while those of the northern states are increased. The structure of the contours of $h_{2}$ is similar for all $Q$ less than a certain $Q_{\text {critical }}(N, \theta, \chi)$.

Finally, returning to Fig. 3, we have plotted in the Stark region a series of classical skeletons of quantum spectra depicting the changes resulting from tilting the fields from perpendicular.

\section{E. Zeeman region: Bifurcations from perpendicular fields near the Zeeman limit}

Spectra in the Zeeman region are also composed of multiple families of states and possess structure which is similar to that we found in the Stark region.

In Fig. 12 we plot the contours of a typical reduced Hamiltonian in the Zeeman region at $Q=0$ and (a) perpendicular fields or (b) near-perpendicular fields.

At perpendicular fields the Hamiltonian is symmetric in $W$ and an $x$-point separatrix divides the contours into three families. In the Zeeman region, the high energy states are equatorial and nondegenerate. In the northern and southern hemispheres are low energy degenerate states for all $Q$ $<Q_{\text {critical }}(N, \theta, \chi)$. Notice that the majority of the phase space is occupied by the nondegenerate equatorial states.

In Fig. 13 we plot a quantum spectrum from the Zeeman region with perpendicular fields. Residing inside the small triangular protrusion are the low energy states. These states are very nearly doubly degenerate with their slight splitting greatest near the classical boundary which divides the spectra in two. All states in the larger turtle-shaped portion of the spectrum are nondegenerate. We identify the states in the small protrusion as symmetric and antisymmetric linear combinations of the northern and southern states. We also identify the nondegenerate states as the equatorial family sepa-

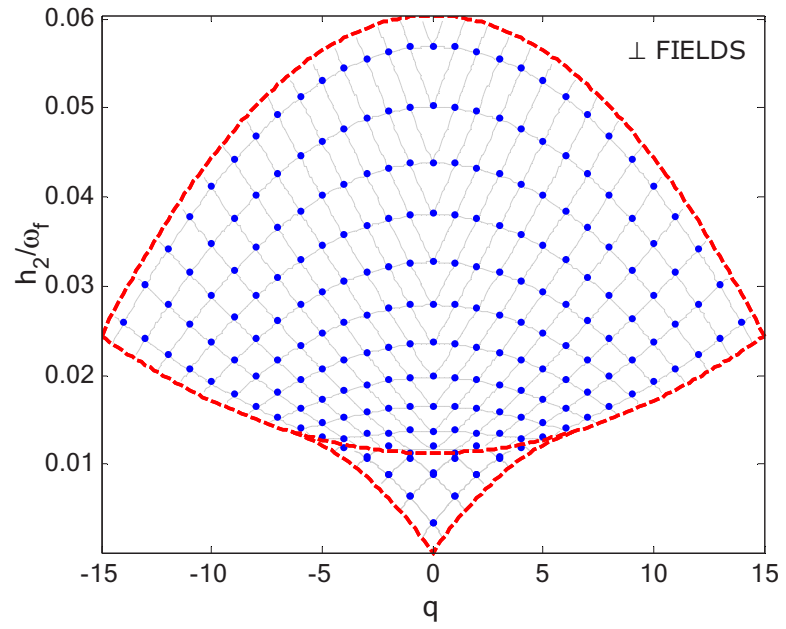

FIG. 13. (Color online) A perpendicular fields spectrum near the Zeeman limit; $h_{2}$ versus $q$ for $\chi=0, n=15, F=39.7 \mathrm{~V} / \mathrm{cm}, B$ $=0.463 \mathrm{~T}$, or $\theta=10^{\circ}$ and $\omega_{f}=10^{-6}$. Spectra with $\chi=0$ and $0<\theta$ $<\theta_{1}$ are composed of two disjoint regular regions. All quantum states in the lower triangular protrusion are doubly degenerate with a slight splitting due to tunneling, while all states in the upper turtle-shaped region are nondegenerate. The boundary between the two regions marks the energy of a saddle point in the reduced second order Hamiltonian $h_{2}$ at each $|q|<Q_{\text {crit }}$.

rated from the nearly degenerate states by the energy of the $x$-point separatrix at each $|q|<Q_{\text {critical }}$.

When the fields are tilted the symmetry is again broken by an additional term proportional to $-\chi W$. In (b) we see that when $\chi$ is increased the northern states acquire a larger energy range and more phase space area while the southern states are depleted of phase space area and span a reduced energy range. Since there is similar structure for all reduced Hamiltonians with $|Q|<Q_{\text {critical }}$, the features of the near perpendicular quantum spectra in the Zeeman region (Fig. 14) are interpreted as follows.

The southern states are confined to the small triangular region bounded by two classical boundaries. The lower boundary marks the minimum energy of the southern contours, while the upper boundary marks the energy of the $x$-point separatrix. The action associated with the southern family is single-valued and smooth, and is depicted by contours (orange online) confined to the small triangular region. The northern family is present in the entire energetically allowed region of the spectrum and the associated action is depicted by gray contours. This action is intrinsically multivalued, as is illustrated in Fig. 14, where lattice vector transport along the gray contours produces an $M_{1}$ defect for any closed loop which encircles the upper boundary of the inner triangle.

\section{CONCLUSION}

We have examined the quantum energy spectrum of hydrogen in weak, nearly perpendicular electric and magnetic fields using both classical and quantum descriptions. We found that the presence of a phenomenon known as monodromy, previously predicted by Sadovskií and Cushman to 


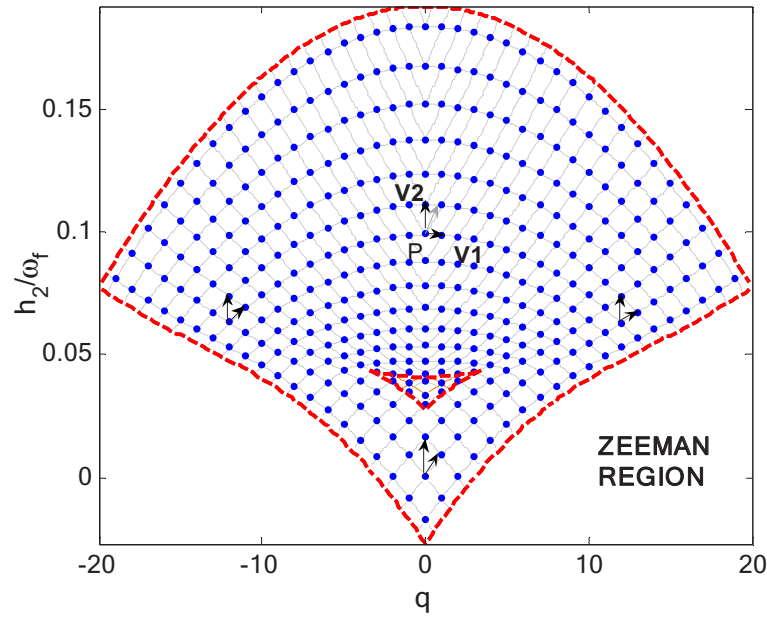

FIG. 14. (Color online) The spectrum $h_{2}$ versus $q$ for $\chi / \omega_{f} n^{3}$ $=1, n=20, F=30 \mathrm{~V} / \mathrm{cm}, B=0.463 \mathrm{~T}$, or $\theta=10^{\circ}$ and $\omega_{f}=10^{-6}$. The quantum basis included $n$ manifolds $18-22$. The triangular region centered about $q=0$ and $h_{2}=0.04$ contains two families of states and the upper boundary acts as a source for a $[1,1 ; 0,1]$ defect. Lattice vector transport of $\left\{\mathbf{V}_{1}, \mathbf{V}_{2}\right\}$ along smooth contours connecting points marked by vector pairs counterclockwise about the degenerate region causes $\mathbf{V}_{1}$ to return to the gray vector $\mathbf{V}_{1}+\mathbf{V}_{2}$. be present in the case of exactly perpendicular fields for a limited range of field strength ratios, not only persists in near perpendicular fields, but assumes new manifestations over a wide range of field ratios. Using classical mechanics we have mapped out the parameter space and found that it is divided into six distinct regions. Each region is identified by a lattice defect structure present in the energy spectrum which is associated with the classical phenomenon known as monodromy. Variation of field strengths and angles leads to transitions between the different defect structures. We have found that a point defect, which was previously predicted to exist at perpendicular fields, undergoes a bifurcation into two distinct point defects as the fields are tilted. We also showed that nonpoint defects arise when fields are tilted from perpendicular near the Stark and Zeeman limits. In a future paper we will present the entire theory in detail.

Note added. Sadovskii and colleagues have recently adapted their framework to consider nonperpendicular fields and obtained results consistent with our findings [16].

\section{ACKNOWLEDGMENTS}

The authors thank Dmitrii Sadovskií for helpful comments and suggestions. This work was supported by the National Science Foundation.
[1] W. Pauli, Z. Phys. 36, 336 (1926). English Translation: B. L. Van der Waerden, Sources of Quantum Mechanic (Dover, New York, 1967).

[2] F. Penent, D. Delande, F. Biraben, and J. C. Gay, Opt. Commun. 49, 184 (1984); G. Wiebusch, J. Main, K. Krüger, H. Rottke, A. Holle, and K. H. Welge, Phys. Rev. Lett. 62, 2821 (1989).

[3] T. P. Grozdanov and E. A. Solov'ev, J. Phys. B 15, 1195 (1982); J. B. Delos, S. K. Knudson, and D. W. Noid, Phys. Rev. A 28, 7 (1983); P. A. Braun, Rev. Mod. Phys. 65, 115 (1993); P. Schmelcher and L. S. Cederbaum, Chem. Phys. Lett. 208, 548 (1993); P. Schmelcher and L. S. Cederbaum, Phys. Rev. A 47, 2634 (1993); E. Flöthmann, J. Main, and K. H. Welge, J. Phys. B 27, 2821 (1994); J. Main and G. Wunner, ibid. 27, 2835 (1994); J. von Milczewski, G. H. F. Diercksen, and T. Uzer, Phys. Rev. Lett. 76, 2890 (1996); J. Main, M. Schwacke, and G. Wunner, Phys. Rev. A 57, 1149 (1998); P. Bellomo, C. R. Stroud, D. Farrelly, and T. Uzer, ibid. 58, 3896 (1998); J. Rao, D. Delande, and K. T. Taylor, J. Phys. B 34, L391 (2001); D. M. Wang and J. B. Delos, Phys. Rev. A 63, 043409 (2001); T. Bartsch, J. Main, and G. Wunner, ibid. 67, 063411 (2003); S. Gekle, J. Main, T. Bartsch, and T. Uzer, ibid. 75, 023406 (2007).

[4] E. A. Solove'v, Sov. Phys. JETP 58, 63 (1983); D. A. Sadovskií and B. I. Zhilinskií, Phys. Rev. A 57, 2867 (1998); N. Berglund and T. Uzer, Found. Phys. 31, 283 (2001).

[5] J. J. Duistermaat, Commun. Pure Appl. Math. 33, 687 (1980); R. Cushman and L. Bates, Global Aspects of Classical Integrable Systems (Birkhauser, Boston, 1997).

[6] In classical integrable systems with $k$ degrees of freedom, there are $k$ functions $F_{i}(p, q)$ which are conserved on every trajectory. Phase space is the union of joint level sets of these functions $\left[\right.$ i.e., $F_{i}(p, q)=f_{i}, i=1 \ldots k$ ] with each level set labeled by the values of the constants of the motion $\left(f_{1}, f_{2}, \ldots, f_{k}\right)$. The space of all allowed values $\left\{f_{i}, i=1 \ldots k\right\}$ is the "classical spectrum." If the functions $F_{i}(p, q)$ are independent at every point in a level set, then each connected component of that level set is a $k$-torus. However, in some systems, certain level sets are not $k$-tori. Those level sets which are $k$-tori may form (a) a single simply connected family, (b) a single multiply connected family, or (c) two or more disjoint families, each of which may be either simply or multiply connected. In a small neighborhood of phase space about any one torus, $k$ smooth action variables may be constructed, providing an alternative labeling for the tori. These smooth action variables are locally single-valued functions of the constants of the motion. However, if a family of tori is multiply connected, smooth continuation of a local action variable may lead to a globally multiplevalued function on the classical spectrum. Whenever smooth continuation of an action variable around some closed loop in the classical spectrum brings that action variable back to a new value, the system is said to have "nontrivial monodromy of action and angle variables," or simply "monodromy."

[7] M. S. Child, J. Phys. A 31, 657 (1988); San Vũ Ngoc, Commun. Math. Phys. 203, 465 (1999); B. I. Zhilinskií, Acta Appl. Math. 87, 281 (2005).

[8] D. A. Sadovskií and R. Cushmann, Physica D 142, 166 (2000).

[9] Nearly perpendicular, weak fields means $|\chi| \lesssim \omega_{f} n^{3} \ll 1$.

[10] The quantum basis must include several $n$ manifolds or impor- 
tant effects, second order in the electric field, will be neglected.

[11] High order classical perturbation indicates that additional structure in a tiny region near the bottom of Fig. 1(b) may prevent globally smooth classical action contours everywhere inside the spectral boundary. See K. Efstathiou, R. Cushman, and D. A. Sadovskií, Physica D 194, 250 (2004).

[12] Equivalently any single choice of action variable may be regarded as a smooth but multivalued function of $q$ and $h_{2}$.

[13] Full details will be published in a separate paper.

[14] Each disjoint energy contour which does not contain a fixed point bisects the $\left(W, \delta_{w}\right)$ phase space area. The area of either division is an appropriate action almost everywhere on the $q h_{2}$ plane. The two choices of locally smooth actions appearing in all plots (save Figs. 1 and 3) are precisely these bisections.

[15] As the energies of the quantum states evolve with $\chi$, avoided crossings occur between northern and southern states within each $q$ cluster.

[16] K. Efstathiou, D. A. Sadovskií, and B. I. Zhilinskií (unpublished). 